\title{
The immune system also gets tired: Overcoming T-cell exhaustion to potentiate anti-cancer immune responses
}

\author{
Emmanuel Bugarin-Estrada* \\ Nuffield Department of Surgical Sciences, University of Oxford, United Kingdom
}

\begin{abstract}
Despite the anti-tumor effects of CD8+ T-cells, chronic exposure to tumor antigens causes T-cell exhaustion, dampening tumor growth control. A subset of exhausted T-cells with stem-like properties ( ${ }_{S L}$ T-cells) has been identified in tumor samples, characterized by the expression of inhibitory molecules (PD-1, CTLA-4), the transcription factor TCF1, and reduced cytotoxic effects.

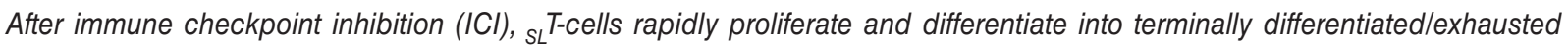
T-cells ${ }_{T D}$ T-cells) with increased effector mechanisms, associating with favorable cancer outcomes patients. Moreover, various epigenetic mechanisms have been implicated in the development of exhausted T-cells, in which the transcription factor TOX has a crucial role. Getting a better understanding of T-cell exhaustion is essential to develop novel prognostic and therapeutic strategies that improve clinical responses to cancer immunotherapies. This review focuses on T-cell exhaustion: features, development, and therapeutic strategies to overcome this dysfunctional T-cell condition in cancer.
\end{abstract}

Key words: T-cell exhaustion. Cancer immunology. Tumor-infiltrating lymphocytes. Stem-like CD8+ T-cells. Immune checkpoint inhibitors.

\section{Introduction}

The immune system plays an active role in recognizing and attacking cancer cells; however, complex interactions between the tumor microenvironment (TME) and immune components exist. Novel therapies to manipulate the immune response against tumors are being implemented with favorable outcomes; nevertheless, several challenges persist ${ }^{1}$.

Particularly, CD8+ T-cells can recognize and kill tumor cells due to their cytotoxic activity. Tumor antigen recognition in the TME is determined by the activation or priming of naive $\mathrm{CD} 8^{+} \mathrm{T}$-cells in the secondary lymphoid organs (SLOS) by cognate antigens bound to
MHC molecules, which form peptide-MHC (pMHC) complexes. The priming process of naïve $\mathrm{CD} 8^{+} \mathrm{T}$-cells is mediated by antigen-presenting cells (APCs), mainly dendritic cells (DCs) ${ }^{2}$. DCs are phagocytic cells that capture tumor antigens in the TME, become activated and then migrate to the SLO (e.g., draining lymph nodes), carrying tumor antigens through pMHC complexes. In the SLO, naïve CD8 ${ }^{+}$T-cells that express T-cell receptors (TCRs) recognizing their cognate antigens become activated by costimulatory molecules and cytokines released by $\mathrm{DCs}{ }^{2}$. Then, primed $\mathrm{CD} 8^{+} \mathrm{T}$-cells migrate to the blood and later infiltrate the TME. These cytotoxic T lymphocytes (CTLs) express TCRs that can recognize tumor antigens presented on the surface of

\section{Correspondence:}

*Emmanuel Bugarin-Estrada

E-mail: emmanuel.bugarin@gmail.com
Available online: $24-12-2020$

Date of reception: 31-10-2020

Date of acceptance: 02-11-2020

DOI: 10.24875/RMU.M20000052
Medicina Universitaria. 2020;22(4):180-187 www.medicinauniversitaria.org

1665-5796/@ 2020 Universidad Autónoma de Nuevo León. Published by Permanyer. This is an open access article under the CC BY-NC-ND license (http://creativecommons.org/licenses/by-nc-nd/4.0/). 
malignant cells through $\mathrm{MHC}$ molecules. After antigen recognition, primed $\mathrm{CD} 8^{+}$T-cells release cytotoxic molecules such as perforins and granzymes that act directly on neoplastic cells ${ }^{3}$.

The anti-tumor effect of CTLs is determined by intrinsic and extrinsic factors in the TME that shape their response against cancer. For example, tumor cells can downregulate MHC molecules' expression, dampening the recognition of tumor antigens by T-cells. In addition, anti-tumor $\mathrm{CD}^{+} \mathrm{T}$-cells may become dysfunctional and tolerant, impairing tumor killing. Different facets of $\mathrm{CD}^{+} \mathrm{T}$-cell dysfunction exist after antigen-specific TCR activation, including anergy, exhaustion, and senescence ${ }^{4,5}$. Some methods to reduce T-cell dysfunction against cancer, including immune checkpoint inhibitors (ICls), induce good clinical outcomes; however, some patients remain resistant to these therapies ${ }^{6}$. Thus, acquiring a better understanding of the immune mechanisms leading to dysfunctional T-cells inside tumors can provide better strategies to kill cancer cells and identify elements that may predict clinical responses. This review focuses on T-cell exhaustion: features, development, and therapeutic strategies to overcome this dysfunctional T-cell condition in cancer.

\section{Tumor-infiltrating lymphocytes (TILs)}

The presence and localization of tumor-infiltrating lymphocytes (TILs) affect the response to immunotherapies and correlate with clinical outcomes in different tumor types. Evidence suggests that cancer patients with high levels of $\mathrm{CD} 8^{+}$TILs survive longer than patients with low levels of $\mathrm{CD}^{+} \mathrm{TILs}^{7,8}$. This might be due to the recruitment of primed $\mathrm{CD} 8^{+} \mathrm{T}$-cells with cytotoxic activity that effectively recognizes and kills target cells. Moreover, patients with immune-inflamed tumors usually respond better to $\mathrm{ICl}$ than patients with immune-excluded tumors ${ }^{9,10}$. These observations might be explained by the reinvigoration of T-cell effector mechanisms against malignancies.

\section{T-cell exhaustion}

Although $\mathrm{CD}^{+}{ }^{+}$T-cells can infiltrate tumor tissues, neoplastic cells and their microenvironment may induce T-cell exhaustion and impair tumor rejection ${ }^{11}$. As explained below, exhausted CD8 ${ }^{+}$TILs share common features with those found in exhausted T-cells associated with chronic viral infections ${ }^{12}$.

\section{Characteristics and development of exhausted T-cells}

Exhausted T-cells are hyporesponsive, terminally differentiated immune cells with limited proliferative potential. They overexpress inhibitory receptors (PD-1, CTLA-4, TIM-3, and LAG-3) and downregulate the expression of costimulatory molecules. Inhibitory receptors on T-cells induce negative effects on their activation and effector function by either: (1) activating intracellular pathways that interfere with signals from the TCR and costimulatory proteins (e.g., CD28), or (2) by competing with ligands that activate costimulatory pathways (e.g., CTLA4 binding on T-cells to CD80/CD86 on DCs) $)^{13,14}$.

Exhausted T-cells were first observed in transgenic CD8 ${ }^{+} \mathrm{T}$-cells bearing lymphocyte choriomeningitis virus (LCMV)-specific TCRs in mice ${ }^{15}$. Further evidence suggested that during chronic LCMV infection, CD8 ${ }^{+} \mathrm{T}$-cells did not have appropriate antiviral effector mechanisms ${ }^{16}$. After acute antigen exposure, naïve $\mathrm{CD}^{+}$ T-cells become activated, differentiating into early effector and memory T-cells. During acute viral infections, early effector T-cells contribute to attacking and controlling infected host cells. In contrast, memory T-cells persist in the circulation and control viral replication if reinfection occurs ${ }^{17}$. However, after prolonged antigen exposure, activated T-cells become hyporesponsive and with limited proliferative potential. Hence, T-cell exhaustion may protect tissues from potentially harmful effects of effector CD8 ${ }^{+} \mathrm{T}^{-}$cells $^{18}$.

In the context of cancer, T-cell exhaustion can be explained by: (1) persistent TCR signaling due to chronic antigen exposure that leads to the sustained expression of inhibitory molecules on T-cells, (2) non-effective interactions with tumor-infiltrating DCs caused by negative costimulatory signals, and (3) due to immunosuppressive effects of cells (e.g., regulatory T-cells) and cytokines (e.g., IL10 and TGF $\beta$ ) in the TME ${ }^{13}$. Thus, strategies to avoid T-cell exhaustion or reactivate exhausted T-cells might induce tumor control.

\section{Effects of ICl on exhausted T-cells}

Blockade of checkpoint molecules has been shown to stimulate anti-tumor T-cell responses and provide clinical benefits in certain types of cancer. Nevertheless, resistance to treatment and the appearance of immune-related adverse events are some of their limitations $^{6}$. Progress is being made to elucidate the mechanisms of $\mathrm{ICl}$ against tumors better. This might 
facilitate the identification of markers of response/ resistance to $\mathrm{ICl}$ and the development of therapeutic strategies to achieve durable clinical responses.

Pauken et al. showed that $\mathrm{ICl}$ induces epigenetic mechanisms that reverse T-cell exhaustion, reacquiring some features of effector T-cells ${ }^{9}$. In contrast, a study in patients with basal cell carcinoma demonstrated that anti-PD1 therapy increased the number of $\mathrm{CD} 8^{+}$TILs due to the expansion of anti-tumor clones that were not present in tumors before ICl. Analysis of TCR sequencing in peripheral blood indicated that, instead of reinvigorating exhausted T-cells, anti-PD1 favored the recruitment of new anti-tumor T-cell clones with different antigen specificity ${ }^{19}$.

Several groups have recently found that $\mathrm{ICl}$ expands a relatively undifferentiated cluster of exhausted $\mathrm{CD}^{+}$ TILs with stem-like properties ${ }^{20,21}$. These studies provided strong evidence that $\mathrm{ICl}$ effects to control tumor growth rely on the expansion of stem-like TILs rather than a reversal of T-cell exhaustion.

\section{Stem-like T-cells and cancer}

Single-cell technologies have enabled scientists to characterize TILs better, clustering them according to their phenotypic and transcriptional profiles. The identification of key elements associated with exhausted T-cells might provide novel biomarkers and therapeutic targets in cancer.

A subset of $P D 1^{+} C D 8^{+}$TILs expressing the transcription factor TCF1 (encoded by the Tcf7 gene) has been identified in melanoma patients, associating with good therapeutic responses after $\mathrm{ICl}$ and with prolonged survival $^{22}$. This population of cells, known as early/progenitor exhausted or stem-like T-cells ( ${ }_{S L}{ }^{T}$-cells), can self-renew and differentiate into terminally exhausted/ terminally differentiated T-cells ( ${ }_{\mathrm{TD}} \mathrm{T}$-cells) ${ }^{21}$. SL T-cells express intermediate levels of PD1, high levels of costimulatory molecules (CD28, ICOS, and SLAMF6), genes associated with survival/proliferation (e.g., IL7R and IL2RA), chemokines (XCL1), and chemokine receptors (CCR7 and CXCR5) that are relevant for T-cell recruitment. However, these $\mathrm{SL}^{\mathrm{T}}$-cells lack additional coinhibitory molecules such as TIM-3 and express low leves of effector molecules (e.g., perforins or granzymes). In contrast, TD T-cells do not express TCF1, have low proliferation potential, and express high levels of PD1, TIM3, LAG3, CD39, and granzymes (Fig. 1).

$\mathrm{SL}^{\mathrm{T}}$-cells have been identified in different tumors, based on the expression of CXCR5 (lung cancer) ${ }^{23}$, or PD1 and TCF1 coexpression (melanoma and kidney cancer) ${ }^{21,24}$, representing a minority of $\mathrm{CD}^{+}$TILs. Similarly, an analogous population of virus-specific $\mathrm{TCF}{ }^{+} \mathrm{T}$-cells with high CXCR5 expression levels has been found in lymphoid tissues during chronic viral infections ${ }^{25}$.

\section{Functional relevance of ${ }_{S L}$ TILs}

Analysis in human tumors has shown that ${ }_{S L} \mathrm{CD} 8^{+}$TILS have more proliferation and differentiation potential than ${ }_{T D} \mathrm{CD}^{+}{ }^{+}$TILs after antigen stimulation ${ }^{24,26}$. Moreover, recent murine studies have demonstrated that ${ }_{S L}{ }^{T}$-cells are important for tumor control, and ICls burst the proliferation of intratumoral ${ }_{\mathrm{SL}} \mathrm{T}$-cells expressing TCF1 and expand the pool of ${ }_{T D}$ T-cells, limiting tumor growth (Fig. 2).

Siddiqui et al. observed that tumor growth was increased in tumor-bearing mice receiving adoptively transferred tumor-specific T-cells lacking TCF1 compared to mice treated with TCF1-expressing T-cells. They found that ${ }_{S L} \mathrm{TILS}\left(\mathrm{TCF} 1^{+} \mathrm{PD} 1^{+}\right)$proliferate and differentiate in response to $\mathrm{ICl}$, increasing the pool of $\mathrm{sL}^{\mathrm{TILs}}$ and ${ }_{\mathrm{TD}} \mathrm{TILS}\left(\mathrm{TCF}^{-} \mathrm{PD} 1^{+}\right)$. Although TCF1 was not essential to generate ${ }_{S L}$ TILS, it was required for ${ }_{S L} \mathrm{TIL}$ proliferation and differentiation ${ }^{21}$.

Similar results were found by Kurtulus et al. They observed that TIM3 and PD1 checkpoint blockade in a colon carcinoma model expanded STILS (PD1-TIM3)). Adoptive transfer of ${ }_{S L}$ TILs into tumor-bearing mice without lymphocytes $\left(\mathrm{RAG}^{-/}\right)$) showed that ${ }_{S L}$ TILs can self-renew and act as precursors of ${ }_{\mathrm{TD}}$ TILS $\left(\mathrm{PD}^{+}{ }^{+} \mathrm{TIM} 3^{+}\right)^{27}$.

In contrast to $\mathrm{SL}_{\mathrm{S}}^{\mathrm{T}}$-cells, exhausted ${ }_{\mathrm{TD}} \mathrm{T}$-cells have been associated with anti-PD1 resistance ${ }^{25}$. However, the blockade of additional pathways in ${ }_{\mathrm{TD}}^{\mathrm{T}}$-cells (e.g., anti-TIM3 and anti-CD39) has been shown to decrease tumor growth and prolong survival in melanoma models ${ }^{22}$. Thus, exhausted SL TILs primarily respond to anti-PD-1 therapy compared to ${ }_{\mathrm{TD}}$ TILs.

Furthermore, it has been shown that ${ }_{\mathrm{SL}}$ TILs associate with improved clinical outcomes. Lung cancer patients responding to $\mathrm{ICl}$ have higher ${ }_{\mathrm{SL}}^{\mathrm{TI}}$ TS in pre-treatment biopsies compared to non-responders ${ }^{23}$. Additional studies showed that an increased ratio of $\mathrm{CD} 8{ }^{+} \mathrm{TCF}^{+}$ to $\mathrm{CD}^{+}{ }^{+} \mathrm{TCF} 1^{-}$- TILs before $\mathrm{ICl}$ predicts better therapeutic responses to anti-PD1 and overall survival in melanoma patients ${ }^{22}$. Similarly, melanoma patients with increased amounts of ${ }_{S L}$ TILs experience a longer duration of response to $\mathrm{ICl}^{20}$. These observations indicate that $\mathrm{ICl}$ is more likely to provide clinical responses in patients who are not able to generate ${ }_{\mathrm{TD}} \mathrm{TILS}$ from $\mathrm{SL}_{\mathrm{T}} \mathrm{TL}$ s before treatment. Thus, strategies to expand and 


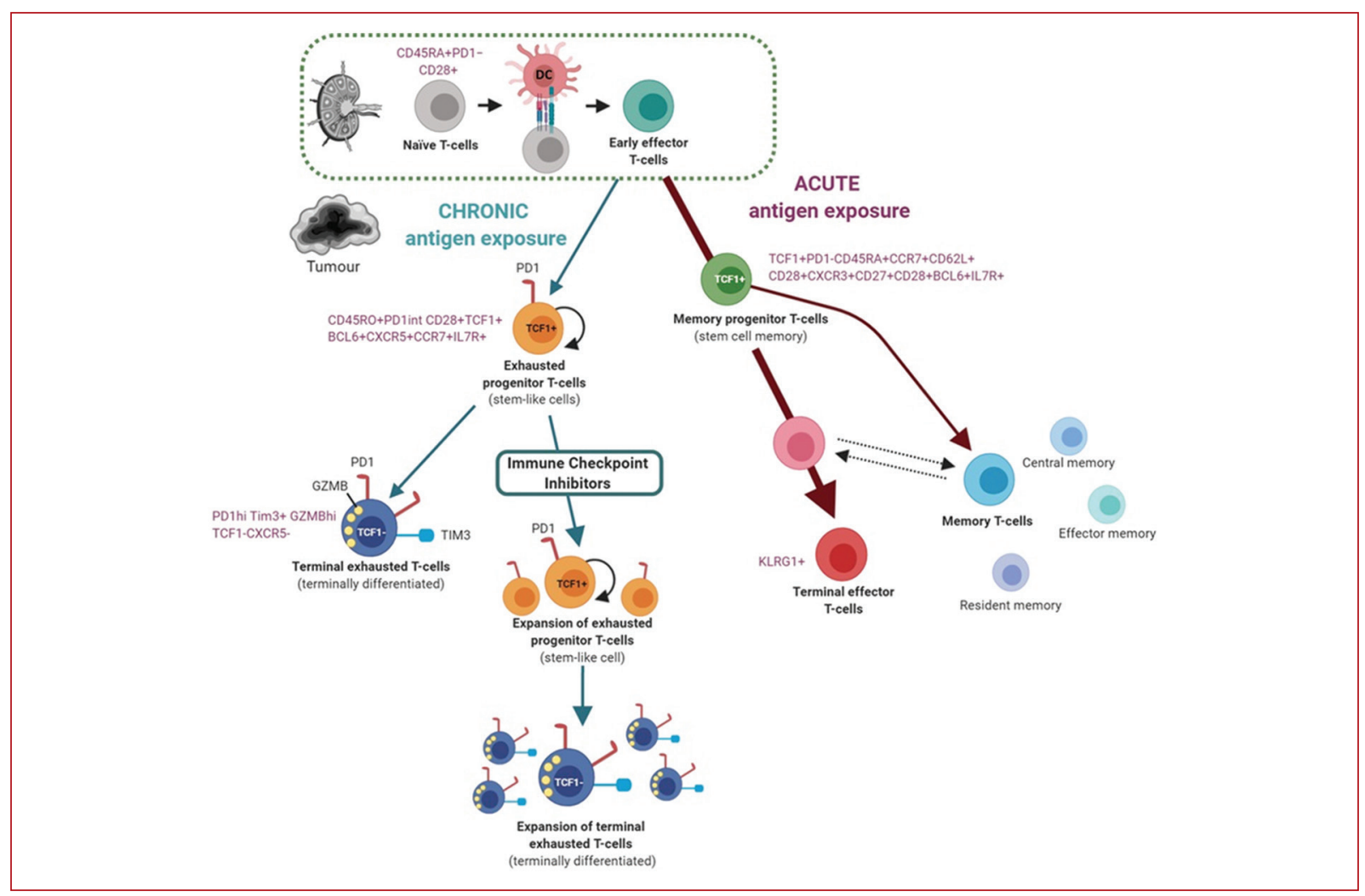

Figure 1. Exhausted progenitor ${ }_{S L}$ T-cells originate after chronic antigen exposure.

It has been recently shown that some tumors have a population of stem-like (SL) CD8+T-cells (TCF1+PD1 int $\left.\mathrm{TIM}^{-} \mathrm{CD} 28^{+}\right)$, able to replicate and differentiate into terminally differentiated (TD) cytotoxic T-cells (TCF1-PD1 ${ }^{\text {hiTIM }}{ }^{+}$CD28 ${ }^{+}$). sL T-cells express high levels of survival genes (e.g., IL7R and IL2RA), costimulatory molecules (CD28, ICOS, and SLAMF6), chemokines (XCL1), and chemokine receptors (CCR7 and CXCR5). In contrast, ${ }_{T D}^{T}$-cells express higher levels of effector molecules (perforins and granzymes) and checkpoint molecules (PD1 and TIM3). Similar populations of $\mathrm{SL}^{\mathrm{T}}$-cells and TDT-cells have also been described during chronic viral infections, suggesting their formation after chronic antigen exposure. Remarkably, exhausted progenitor ${ }_{S L}^{T}$-cells share similar features with memory progenitor T-cells $\left(\mathrm{PD} 1^{-} \mathrm{TCF} 1^{+}\right)$, including the increased expression of Lef1, Bcl6, and IL7R. Memory progenitor T-cells develop after acute antigen exposure and facilitate the differentiation and persistence of memory $\mathrm{CD}^{+}$T-cells. | Figure created in ${ }^{\circledR}$ BioRender - biorender.com.

preserve ${ }_{S L}$ TILs might represent an important approach to improve anti-tumor immune responses.

\section{$\mathrm{SL}^{\mathrm{T}}$-cells and APC-enriched niches}

Recent studies have identified a subpopulation of ${ }_{S L} \mathrm{CD} 8^{+}$TILs that preferentially locate within MHC-II dense regions inside the stromal barrier of human tumors (APC niches) ${ }^{24}$, which also contain blood and lymphatic vessels (Fig. 3). The presence of APC niches correlates with the number of CD8 ${ }^{+}$TILs, associating with good outcomes in cancer patients ${ }^{24}$ and improved responses to $\mathrm{ICl}^{20,22}$. This is consistent with the requirement of CD28 costimulation to promote $\mathrm{PD} 1^{+} \mathrm{CD} 8^{+} \mathrm{T}$-cell expansion after $\mathrm{ICl}^{28}$.
It has been suggested that these APC niches might protect ${ }_{\mathrm{SL}}$ TILs from exposure to high levels of tumor antigens and provide costimulatory signaling to enhance ${ }_{S L}$ TIL proliferation and differentiation in response to $\mathrm{ICl}^{29}$. In contrast, ${ }_{\mathrm{TD}}^{\mathrm{T}} \mathrm{T}$-cells preferentially interact with tumor cells, and $\mathrm{ICI}$ may enhance their cytotoxic effects.

\section{Regulation of ${ }_{S L}$ and ${ }_{T D} T$-cell programming as a therapeutic strategy against cancer}

Epigenetic modifications during the differentiation process of exhausted T-cells can determine the potential of ${ }_{S L}$ TILs to exert sustained anti-tumor responses ${ }^{30}$. 


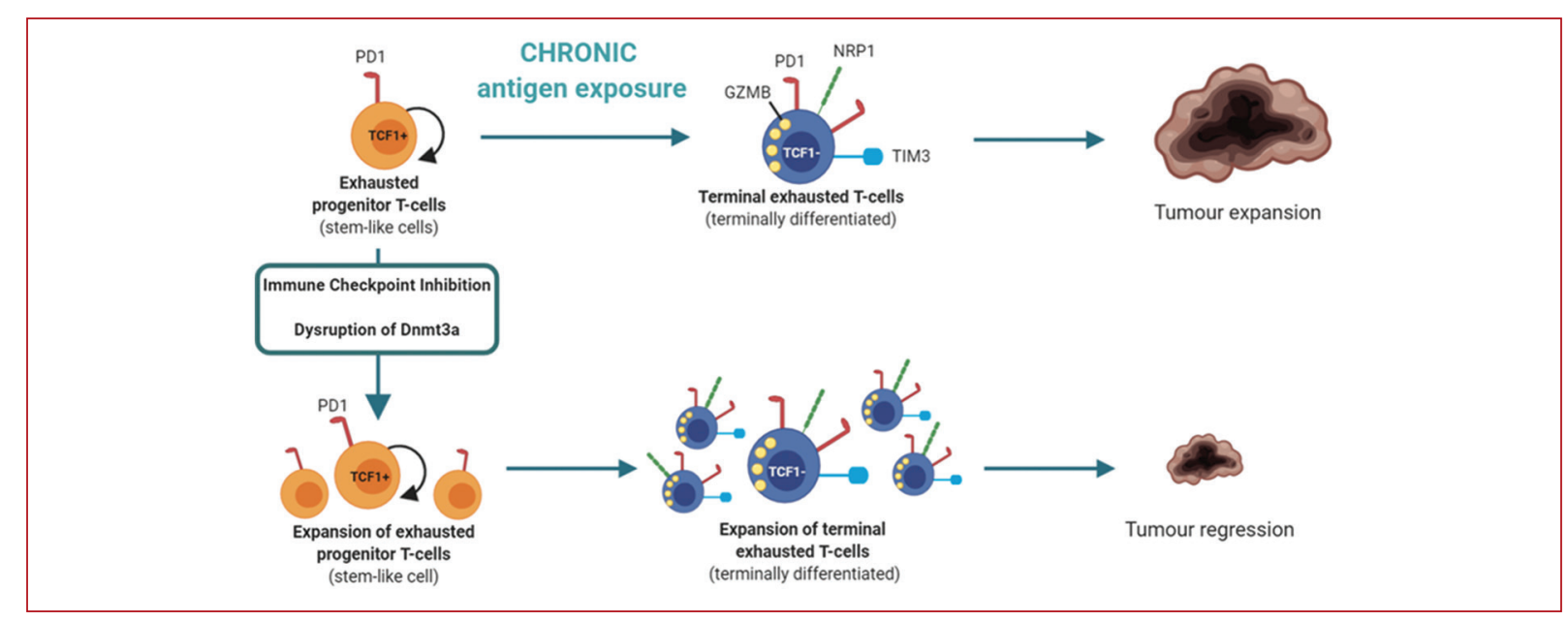

Figure 2. Immune checkpoint inhibitors and modification of epigenetic mechanisms expand the pool of stem-like and terminally differentiated T-cells in tumors.

Available evidence suggests that after priming, early effector T-cells infiltrate non-lymphoid tissues, where they become exhausted after chronic antigen exposure. However, recent studies in humans and mice indicate that undifferentiated tumor-specific ${ }_{S L} \mathrm{CD}^{+}$T-cells can infiltrate the TME, acquiring exhaustion features and persist within tumor tissue. Here, tumor-specific ${ }_{S L}$ T-cells expand and differentiate into TDT-cells in response to ICI, increasing the amount of tumor-specific T-cells. Subsequently, ${ }^{\mathrm{TD}} \mathrm{CD} 8^{+} \mathrm{T}$-cells migrate and interact with tumor cells, where anti-PD1 will enhance their effector functions, contributing to tumor regression. Moreover, inhibition of DNA methylation (e.g., disruption of DNA methyltransferase 3 alpha; DNMT3A) in combination with $\mathrm{ICl}$ enhances the proliferation of tumor-specific CD8 ${ }^{+}$T-cells and polyclonal PD1 ${ }^{\text {hi }}$ TILs, causing significant control of tumor growth. | Figure created in ${ }^{\circledR}$ BioRender - biorender.com.

\section{Exhaustion induced by TOX}

TOX is a transcription factor that activates epigenetic programs associated with $\mathrm{CD} 8^{+} \mathrm{T}$-cell exhaustion. TOX is induced through calcineurin/NFAT after persistent TCR stimulation, causing chromatin remodeling, overexpression of inhibitory receptors, and reduction of cytokine production ${ }^{31}$.

It has been shown that TILs from cancer patients express high levels of TOX, correlating with the expression of exhaustion markers (PD1 and TIM3) ${ }^{32}$. Moreover, the loss of TOX in tumor-specific CD8 ${ }^{+}$T-cells failed to persist in tumors and remained dysfunctional even when the exhaustion program was abrogated (i.e., cells showed high expression of TCF1 and inhibitory receptors were not upregulated) ${ }^{32}$. Another study confirmed the lack of ${ }_{T D}$ T-cell responses when TOX was completely depleted; however, the transfer of partially TOX-deficient $\left(\mathrm{TOX}^{+-}\right)$T-cells into tumor-bearing mice reduced tumor growth ${ }^{31}$. Moreover, a variable correlation between TOX and TCF1 has been observed ${ }^{31,32}$. Thus, partially reducing TOX activity during adoptive cell transfer might reduce exhaustion without decreasing the number of functional T-cells within tumors; however, further studies are required to determine this effect.

\section{Exhaustion-associated DNA methylation programs}

Furthermore, the acquisition of de novo DNA methylation has been identified in exhausted $\mathrm{CD}^{+}{ }^{+}$TILs. Ghoneim et al. observed new methylated regions (sup-

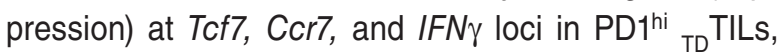

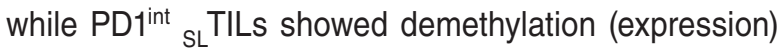
of Ccr7 and Tcf7 loci, suggesting that de novo DNA methylation at specific loci induces terminal differentiation of exhausted T-cells ${ }^{33}$. Moreover, these exhaustion-associated epigenetic programs were preserved in the progeny of ${ }_{\mathrm{TD}}$ TILs and could not be modified after anti-PD-1 monotherapy in cancer patients ${ }^{33}$. However, inhibition of DNA methylation using decitabine combined with $\mathrm{ICl}$ induced the proliferation of polyclonal PD1 ${ }^{\text {hi }}$ TILs and tumor-specific CD8+ T-cells within the TME, causing significant control of tumor growth ${ }^{33}$. Thus, differences in ${ }_{S L}$ and ${ }_{T D}$ T-cells associate with distinct DNA methylation programs, which could be manipulated to improve ICls effect on cancer patients. 


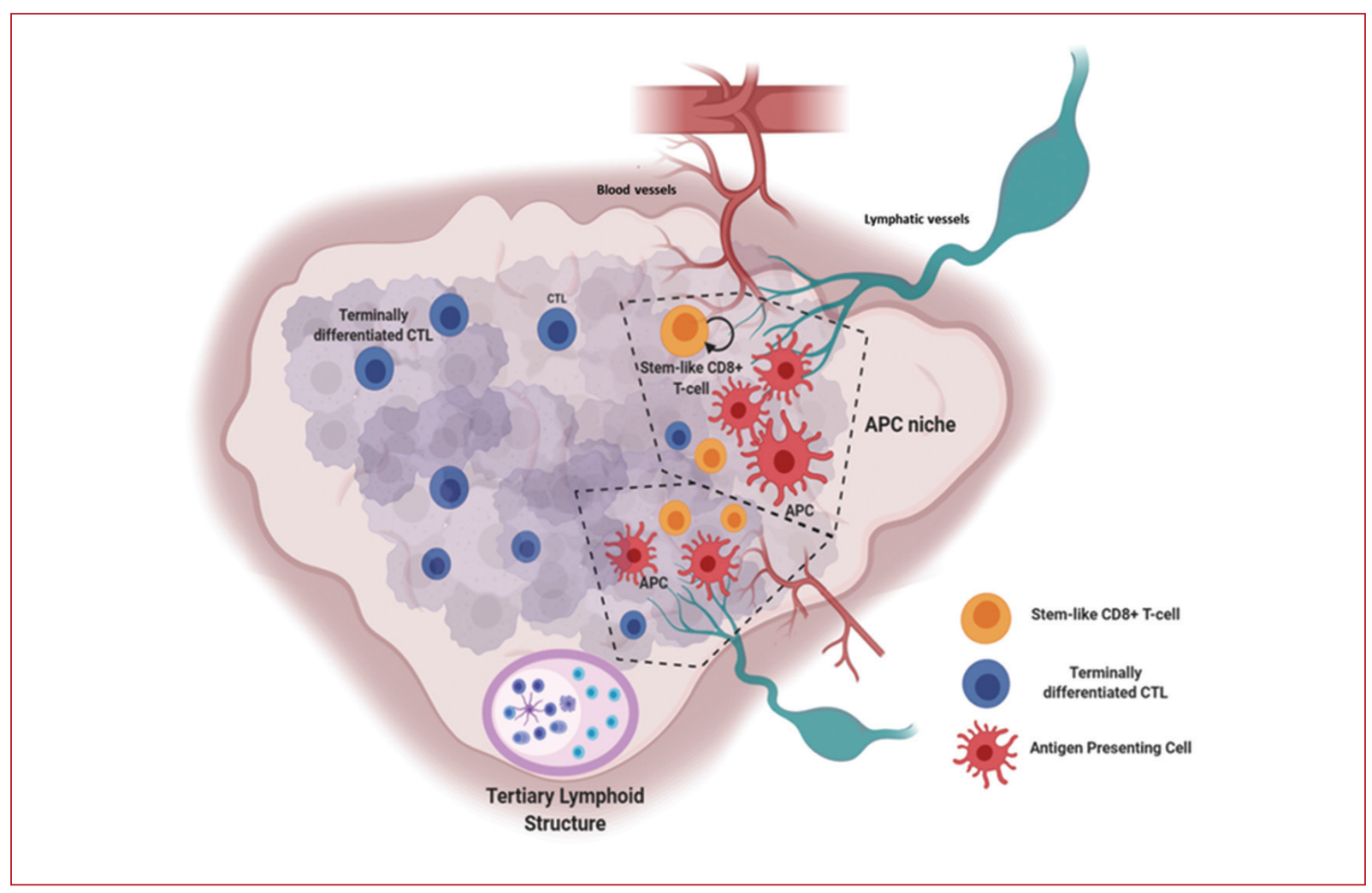

Figure 3. Antigen-presenting cell (APC) niches inside tumors appear to maintain stem-like T-cell proliferation and differentiation into more terminally differentiated effector T-cells. Created using data from Jansen CS, et al. ${ }^{24}$

Evidence indicates that ${ }_{\mathrm{SL}} \mathrm{T}$-cells are present in immune-inflamed tumors, which have increased numbers of ${ }_{\mathrm{TD}} \mathrm{T}$-cells. In contrast, tumors with poor CD8 ${ }^{+}$T-cell infiltration have fewer ${ }_{T D}{ }^{T}$-cells, while ${ }_{S L}{ }^{T}$-cells were still present, suggesting that ${ }_{S L}{ }^{T}$-cells cannot differentiate into ${ }_{T D}$ T-cells in immune-dessert tumors. Further analysis has shown that ${ }_{S L}{ }^{T}$-cells preferentially locate within MHC-II dense regions inside the stromal barrier of tumors (APC niches), which also contain blood and lymphatic vessels. In contrast, tertiary lymphoid structures (TLSs) locate outside the stromal barrier and do not correlate with the number of $\mathrm{CD}^{+}$TILs. Immunofluorescence analyses have shown that APC niches closely resemble extrafollicular regions of lymphoid tissue, while TLS looks more like follicular regions. Furthermore, the presence of APC niches correlates with the number of $C D 8^{+}$TILs and inversely correlates with tumor progression in cancer patients. Evidence also suggests that CD28 costimulation is needed to induce the proliferation and differentiation of ${ }_{S L}$ T-cells after anti-PD1 therapy. Thus, APCs expressing CD80/CD86 might facilitate $\mathrm{SL}^{\mathrm{T}}$-cell differentiation and protect them from chronic exposure to tumor antigens. In response to anti-PD1, APCs might provide costimulatory signaling to enhance ${ }_{S L}{ }^{T}$-cell proliferation and differentiation to ${ }_{\mathrm{TD}} \mathrm{T}$-cells. In contrast, ${ }_{T D} \mathrm{~T}$-cells preferentially interact with tumor cells, and ICI enhances their cytotoxic effects. | Figure created in ${ }^{\oplus}$ BioRender - biorender.com.

\section{Decreasing CAR T-cell exhaustion in solid tumors}

Modified T-cells expressing chimeric antigen receptors (CAR T-cells) have been effective against advanced hematological malignancies because they target cancer antigens that do not require peptide binding MHC molecules. However, these therapies' success in solid tumors has been limited due to several factors, including exhaustion ${ }^{34}$. Increasing the effector and memory functions of CAR T-cells can promote their persistence and improve their anti-tumor response. This has been shown in preclinical models using CAR T-cells with a stem-like memory phenotype ${ }^{35,36}$.

Stem-like cells can be induced from naïve T-cells by triggering Wnt signaling using GSK3 $\beta$ inhibitors ${ }^{37}$, or after their activation with anti-CD3/CD28 antibodies and the addition of IL7 and IL15 in vitro ${ }^{38}$. IL15 can also expand human CXCR5 ${ }^{+} \mathrm{CD} 8^{+}$TILs ex vivo, preserving their stem-like properties compared to IL2 ${ }^{26}$.

CAR T-cells stimulated with anti-CD3/CD28 and cultured with IL7 and IL15 can promote a stem-like phenotype with low expression of exhaustion markers 
(PD1/LAG3), increased cytotoxicity, proliferation, and survival ${ }^{36}$. Similarly, the adoptive transfer of $\mathrm{CXCR}^{+}{ }^{-\mathrm{TIM}^{-}}{ }_{\mathrm{SL}} \mathrm{TILS}$ has been shown to promote tumor control in tumor-bearing mice ${ }^{39}$. Thus, generating CAR T-cells with stem-like properties are a promising strategy to enhance anti-tumor effects and improve their expansion and survival in solid tumors.

\section{Conclusions and perspectives}

T-cell exhaustion represents a major limitation to anti-tumor immune responses. $\mathrm{ICI}$ has been shown to enhance cytotoxic effects against malignant cells. One of these mechanisms involves the expansion of the pool of stem-like and terminally differentiated T-cells in the TME. Thus, manipulating epigenetic programs in T-cells to prevent or revert exhaustion might represent a potential therapeutic option in cancer. For example, modified T-cells with stem-like properties could be genetically engineered to express CARs. It can be suggested that CARs developed using reprogrammed T-cells with stem-like properties can provide sustained immune responses against solid tumors due to their proliferative and differentiation capacities into cells with effector functions and the reduced expression of exhaustion markers. The characterization and functional evaluation of $\mathrm{SL}_{\mathrm{TILS}}$ to develop and test CAR constructs in preclinical models might determine the possibility to translate these approaches into clinical studies.

Future work will be required to identify intracellular pathways associated with coinhibitory molecules that cause exhaustion, such as TIM3, and to elucidate the mechanisms by which single or combined ICI strategies work. Identifying the mechanisms by which APC niches are formed in tumors and how do different tumor types interfere with their formation is also needed. Stimulating the growth of these niches might enhance the preservation of $\mathrm{SL}^{\mathrm{T}}$-cells or CAR T-cells within tumor tissue, improve the anti-tumor immune responses of $\mathrm{ICl}$, and increase T-cell infiltration to the TME. Moreover, work to standardize the identification of SL TILS using different approaches will be necessary to optimize their clinical applicability.

\section{Acknowledgments}

The author wants to acknowledge the financial support received by CONACYT for his postgraduate studies.

\section{Conflicts of interest}

The author declares that they have no conflicts of interest.

\section{Ethical disclosures}

Protection of human and animal subjects. The authors declare that no experiments were performed on humans or animals for this study.

Confidentiality of data. The authors declare that no patient data appear in this article.

Right to privacy and informed consent. The authors declare that no patient data appear in this article.

\section{References}

1. Hegde PS, Chen DS. Top 10 challenges in cancer immunotherapy. Immunity. 2020;52:17-35.

2. Noubade R, Majri-Morrison S, Tarbell KV. Beyond CDC1: emerging roles of DC crosstalk in cancer immunity. Front Immunol. 2019;10:1014.

3. Waldman AD, Fritz JM, Lenardo MJ. A guide to cancer immunotherapy: from $T$ cell basic science to clinical practice. Nat Rev Immunol. 2020; 20:651-68.

4. EITanbouly MA, Noelle RJ. Rethinking peripheral T cell tolerance: checkpoints across a T cell's journey. Nat Rev Immunol. 2020. In press. doi: 10.1038/s41577-020-00454-2

5. Zhao Y, Shao Q, Peng G. Exhaustion and senescence: two crucial dysfunctional states of $\mathrm{T}$ cells in the tumor microenvironment. Cell Mol Immunol. 2020;17:27-35.

6. Jenkins RW, Barbie DA, Flaherty KT. Mechanisms of resistance to immune checkpoint inhibitors. Br J Cancer. 2018;118:9-16.

7. Galon J, Costes A, Sanchez-Cabo F, Kirilovsky A, MlecnikB, Lagorce-Pagès C et al. Type, density, and location of immune cells within human colorectal tumors predict clinical outcome. Science. 2006; 313:1960-4.

8. Sato E, Olson SH, Ahn J, Bundy B, Nishikawa H, Qian F, et al. Intraepithelial CD8+ tumor-infiltrating lymphocytes and a high CD8+/regulatory $T$ cell ratio are associated with favorable prognosis in ovarian cancer. Proc Natl Acad Sci U S A. 2005;102:18538-43.

9. Pauken KE, Sammons MA, Odorizzi PM, Manne S, Godec J, Khan O, et al. Epigenetic stability of exhausted T cells limits durability of reinvigoration by PD-1 blockade. Science 2016;354:1160-5.

10. Herbst RS, Soria JC, Kowanetz M, Fine GD, Hamid O, Gordon MS, et al. Predictive correlates of response to the anti-PD-L1 antibody MPDL3280A in cancer patients. Nature. 2014;515:563-7.

11. Willimsky G, Blankenstein T. Sporadic immunogenic tumours avoid destruction by inducing T-cell tolerance. Nature. 2005;437:141-6.

12. Paley MA, Kroy DC, Odorizzi PM, Johnnidis JB, Dolfi DV, Barnett BE, et al. Progenitor and terminal subsets of CD8+ T cells cooperate to contain chronic viral infection. Science. 2012;338:1220-5.

13. McLane LM, Abdel-Hakeem MS, Wherry EJ. CD8 T cell exhaustion during chronic viral infection and cancer. Annu Rev Immunol. 2019;37:457-95.

14. Schietinger A, Philip M, Krisnawan VE, Chiu EY, Delrow JJ, Basom RS, et al. Tumor-specific $T$ cell dysfunction is a dynamic antigen-driven differentiation program initiated early during tumorigenesis. Immunity. 2016; 45:389-401.

15. Moskophidis D, Lechner F, Pircher H, Zinkernagel RM. Virus persistence in acutely infected immunocompetent mice by exhaustion of antiviral cytotoxic effector T cells. Nature. 1993;362:758-61.

16. Zajac AJ, Blattman JN, Murali-Krishna K, Sourdive DJ, Suresh M, Altman JD, et al. Viral immune evasion due to persistence of activated T cells without effector function. J Exp Med. 1998;188:2205-13.

17. Wherry EJ. T cell exhaustion Nat Immunol. 2011:12:492-9.

18. Hossain MA, Liu G, Dai B, Si Y, Yang Q, Wazir J, et al. Reinvigorating exhausted CD8+ cytotoxic T lymphocytes in the tumor microenvironment and current strategies in cancer immunotherapy. Med Res Rev. 2020. In press. doi: https://doi.org/10.1002/med.21727

19. Yost KE, Satpathy AT, Wells DK, Qi Y, Wang C, Kageyama R, et al. Clonal replacement of tumor-specific T cells following PD-1 blockade. Nat Med. 2019;25:1251-9.

20. Miller BC, Sen DR, Al Abosy R, Bi K, Virkud YV, LaFleur MW, et al Subsets of exhausted CD8(+) T cells differentially mediate tumor control and respond to checkpoint blockade. Nat Immunol. 2019;20:326-36. 
21. Siddiqui I, Schaeuble K, Chennupati V, Marraco SA, Calderon-Copete S, Ferreira DP, et al. Intratumoral Tcf1 + PD-1 + CD8 + T cells with stem-like properties promote tumor control in response to vaccination and checkpoint blockade immunotherapy. Immunity. 2019;50:195-211.e10.

22. Sade-Feldman M, Yizhak K, Bjorgaard SL, Ray JP, de Boer CG, Jenkins RW, et al. Defining $T$ cell states associated with response to checkpoint immunotherapy in melanoma. Cell. 2018;175:998-1013.e20.

23. Thommen DS, Koelzer VH, Herzig P, Roller A, Trefny M, Dimeloe S, et al. A transcriptionally and functionally distinct $\mathrm{pd}-1+\mathrm{cd} 8+\mathrm{t}$ cell pool with predictive potential in non-small-cell lung cancer treated with $\mathrm{pd}-1$ blockade. Nat Med. 2018;24:994-1004.

24. Jansen CS, Prokhnevska N, Master VA, Sanda MG, Carlisle JW Bilen MA, et al. An intra-tumoral niche maintains and differentiates stem-like CD8 T cells. Nature. 2019:576:465-470.

25. Im SJ, Hashimoto M, Gerner MY, Lee J, Kissick HT, Burger MC, et al. Defining CD8+ T cells that provide the proliferative burst after PD-1 therapy. Nature. 2016;537:417-21.

26. Brummelman J, Mazza EM, Alvisi G, Colombo FS, Grilli A, Mikulak A et al. High-dimensional single cell analysis identifies stem-like cytotoxic CD8+ T cells infiltrating human tumors. J Exp Med. 2018;215:2520-35.

27. Kurtulus S, Madi A, Escobar G, Klapholz M, Nyman J, Christian E, et al. Checkpoint blockade immunotherapy induces dynamic changes in PD-1 CD8 + tumor-infiltrating T cells. Immunity. 2019;50:181-94.e6.

28. Kamphorst AO, Wieland A, Nasti T, Yang A, Zhang R, Barber DL, et al Rescue of exhausted CD8 T cells by PD-1-targeted therapies is CD28-dependent. Science. 2017;355:1423-7.

29. Hashimoto M, Kamphorst AO, Im SJ, Kissick HT, Pillai RN Ramalingam SS, et al. CD8 T cell exhaustion in chronic infection and cancer: opportunities for interventions. Annu Rev Med. 2018;69:301-18.
30. Philip M, Fairchild L, Sun L, Horste EL, Camara S, Shakiba M, et al. Chromatin states define tumour-specific $\mathrm{T}$ cell dysfunction and reprogramming. Nature. 2017;545:452-6.

31. Khan O, Giles JR, McDonald S, Manne S, Ngiow SF, Patel KP, et al. TOX transcriptionally and epigenetically programs CD8+ T cell exhaustion. Nature. 2019;571:211-8.

32. Scott AC, Dündar F, Zumbo P, Chandran SS, Klebanoff CA, Shakiba M, et al. TOX is a critical regulator of tumour-specific T cell differentiation. Nature. 2019;571:270-4.

33. Ghoneim HE, Fan Y, Moustaki A, Abdelsamed HA, Dash P, Dogra P, et al. De novo epigenetic programs inhibit PD-1 blockade-mediated T cell rejuvenation. Cell. 2017;170:142-57.e19.

34. Choe JH, Williams JZ, Lim WA. Engineering T cells to treat cancer: the convergence of immuno-oncology and synthetic biology. Annu Rev Cancer Biol. 2020;4:121-39.

35. Xu Y, Zhang M, Ramos CA, Durett A, Liu E, Dakhova O, et al. Closely related T-memory stem cells correlate with in vivo expansion of CAR.CD19-T cells and are preserved by IL-7 and IL-15. Blood. 2014;123:3750-9.

36. Gargett T, Brown MP. Different cytokine and stimulation conditions influence the expansion and immune phenotype of third-generation chimeric antigen receptor T cells specific for tumor antigen GD2. Cytotherapy. 2015;17:487-95.

37. Gattinoni L, Lugli E, Ji Y, Pos Z, Paulos CM, Quigley MF, et al. A human memory T cell subset with stem cell-like properties. Nat Med. 2011;17:1290-7.

38. Cieri N, Camisa B, Cocchiarella F, Forcato M, Oliveira G, Provasi E, et al. IL-7 and IL-15 instruct the generation of human memory stem $T$ cells from naive precursors. Blood. 2013;121:573-84.

39. Vodnala SK, Eil R, Kishton RJ, Sukumar M, Yamamoto TN, Ha NH, et al. $\mathrm{T}$ cell stemness and dysfunction in tumors are triggered by a common mechanism. Science. 2019;363:0135. 